\title{
Abordagem orto-preventiva de perda precoce do segundo molar decíduo: relato de
}

\author{
caso \\ Ortho-preventive approach to early loss of the deciduous second molar: case report \\ Abordaje ortopreventivo de la pérdida temprana del segundo molar temporal: reporte de caso
}

Recebido: 08/12/2021 | Revisado: 13/12/2021 | Aceito: 13/12/2021 | Publicado: 21/12/2021

David Bruno Abdon Fernandes

ORCID: https://orcid.org/0000-0002-2710-8404

Centro Universitário Fametro, Brasil

E-mail: brunodavid838@gmail.com

Maria Victória de Araújo Sampaio

ORCID: https://orcid.org/0000-0001-8231-5302 Centro Universitário Fametro, Brasil

E-mail: mariavictoria_sampaio@hotmail.com

Gabriela de Figueiredo Meira

ORCID: https://orcid.org/0000-0002-8285-8769

Universidades Federal de Santa Maria, Brasil E-mail: gabrielameira1@hotmail.com

José Ricardo Prando dos Santos ORCID: https://orcid.org/0000-0002-3044-6383 Universidade Estadual Paulista, Brasil E-mail: jrpsantos@uea.edu.br

Bruna Ramos Meireles dos Santos ORCID: https://orcid.org/0000-0002-3796-4925 Universidade Estadual Paulista, Brasil E-mail: bruna.maisonoral@gmail.com

\begin{abstract}
Resumo
Objetivo: O objetivo deste trabalho foi recuperar um espaço dentário perdido em decorrência de perda precoce do segundo molar decíduo. Na abordagem foi utilizado um recuperador de espaço removível e finalizado com aparelho fixo segmentado. Como resultado foi observado a recuperação total do espaço e irrupção físiológica do pré-molar impactado, ressaltando a importância da ortodontia preventiva e interceptativa na correção de má-oclusão diagnosticada precocemente. Metodologia: Partindo da permissão da paciente por meio do Termo de Consentimento Livre e Esclarecido em partilhar sua imagem para devido fim, o presente estudo trata de um relato de caso clínico de cunho analítico descritivo (Pereira et al., 2018). Relato de caso: Paciente, sexo masculino, 9 anos de idade apresentava má-oclusão classe II dentária direita subdivisão 1 de Angle, devido a perda precoce do elemento 55 (segundo molar superior direito decíduo). O tratamento proposto foi a instalação de um aparelho recuperador de espaço removível, sendo necessário sua substituição ao longo do tratamento por um aparelho fixo segmentado com mola digital em decorrência da pandemia de COVID-19, a fim acelerar a recuperação do espaço e aproveitar a maior força eruptiva do elemento dentário. Conclusão: Os recursos ofertados com o uso de aparelhos recuperadores de espaço são eficazes para interceptar e tratar más-oclusões diagnosticadas precocemente.
\end{abstract}

Palavras-chave: Ortodontia; Má-oclusão; Recuperador de espaço.

\begin{abstract}
Objective: The objective of this work was to recover a lost tooth space as a result of early loss of the second primary molar. In the approach, a removable space stove was used and finished with segmented fixed appliance. As a result, full recovery of space and physiological eruption of the impacted premolar were observed, emphasizing the importance of preventive and interceptive orthodontics in the correction of early diagnosed malocclusion. Methodology: Based on the patient's permission through the Free and Informed Consent Term to share their image for the proper purpose, this study is a clinical case report of descriptive analytical nature (Pereira et al., 2018). Case report: A 9-year-old male patient presented with a Class II right dental subdivision 1 malocclusion of Angle, due to early loss of element 55 (deciduous upper right second molar). The treatment proposed the installation of a removable space recovery device, which was necessary to replace it throughout the treatment with a segmented fixed device with a finger clip due to the COVID-19 pandemic, in order to accelerate the recovery of the space and take advantage of the greater eruptive force of the dental element. Conclusion: The resources offered with the use of space recovery devices are effective in intercepting and treating early diagnosed malocclusions.
\end{abstract}

Keywords: Orthodontics; Malocclusion; Space retriever. 


\begin{abstract}
Resumen
Objetivo: El objetivo de este trabajo fue recuperar un espacio dentario perdido como consecuencia de la pérdida temprana del segundo molar temporal. En el abordaje se utilizó una estufa extraíble y se terminó con un aparato fijo segmentado. Como resultado, se observó la recuperación total del espacio y la erupción fisiológica del premolar impactado, enfatizando la importancia de la ortodoncia preventiva e interceptiva en la corrección de la maloclusión diagnosticada precozmente. Metodología: Con base en el permiso del paciente a través del Término de Consentimiento Libre e Informado para compartir su imagen con el propósito adecuado, este estudio es un reporte de caso clínico de naturaleza analítica descriptiva (Pereira et al., 2018). Caso clínico: Un paciente de 9 años de edad presentó una maloclusión de Angle subdivisión 1 dentaria derecha clase II debido a la pérdida temprana del elemento 55 (segundo molar superior derecho temporal). El tratamiento planteó la instalación de un dispositivo de recuperación de espacio extraíble, que fue necesario sustituirlo durante todo el tratamiento por un dispositivo fijo segmentado con pinza de dedo debido a la pandemia de COVID-19, con el fin de acelerar la recuperación del espacio y aprovechar de la mayor fuerza eruptiva del elemento dentario. Conclusión: Los recursos que se ofrecen con el uso de dispositivos de recuperación de espacio son efectivos para interceptar y tratar maloclusiones diagnosticadas de manera temprana.

Palabras clave: Ortodoncia; Maloclusión; Perro perdiguero del espacio.
\end{abstract}

\title{
1. Introdução
}

Os elementos decíduos desempenham papel importante na mastigação, normoclusão, fala e preservação de espaço para os dentes sucessores permanentes, por isso a perda precoce desses elementos pode ser a causa de atividades parafuncionais, deficiências mastigatórias e fonéticas, alteração no comportamento psicossocial e instabilidade do sistema estomatognático, além de favorecer a instalação de más-oclusões (Monte-Santo et al., 2018; Amaral et al., 2019)

A cárie é o principal fator etiológico responsável pelas perdas precoces dos dentes decíduos, é considerada como a doença crônica mais prevalente em crianças, afeta cerca de $5 \%$ a $20 \%$ das crianças na faixa etária de 01 a 03 anos de idade Godoi et al., 2019; Neto et al., 2016). Possui origem complexa e envolve inúmeros fatores, como: dieta, condição socioeconômica e qualidade de higiene bucal (López et al., 2020; Félez et al., 2020)

Atrás apenas da cárie dentária e doença periodontal, se encontram as más-oclusões que ocupam o terceiro lugar como problema de saúde (Morais et al., 2016; Lopes et al., 2019). Podem induzir negativamente o desempenho de funções do sistema estomatognático e comprometer o desenvolvimento psicossocial, uma vez que a estética é afetada (Rosa et al., 2015; Barbosa et al., 2018).

Através da ortodontia preventiva e interceptativa é possível realizar o diagnóstico prévio de hábitos bucais considerados incorretos que podem alterar o desenvolvimento normal da oclusão. Além disso, este ramo da ortodontia tem ação fundamental na preservação de espaço de dentes decíduos perdidos precocemente, com o uso dos aparelhos mantenedores de espaço (Gomes et al., 2020; Macedo et al., 2015).

No entanto, quando o dente já foi perdido há algum tempo e houve perda de espaço significativa, são indicados os recuperadores de espaço que atuam restabelecendo o espaço perdido, possibilitando que o sucessor permanente possa erupcionar corretamente (Siqueira et al., 2015; Moreno et al., 2018).

Portanto, o objetivo do presente estudo foi relatar um caso de perda precoce do segundo molar decíduo, através do aparelho recuperador de espaço e demonstrar a importância da ortodontia preventiva e interceptativa na correção de más oclusões diagnosticada precocemente.

\section{Metodologia}

Partindo da permissão da paciente por meio do Termo de Consentimento Livre e Esclarecido em partilhar sua imagem para devido fim, o presente estudo trata de um relato de caso clínico de cunho analítico descritivo (Pereira et al., 2018) sobre fechamento de diastemas através da técnica direta de resina composta, realizado na clínica odontológica do CEUNI Fametro, 
na disciplina de estágio supervisionado em Clínica Integrada III, do curso de graduação em odontologia do Centro Universitário Fametro.

\section{Resultados e Discussão}

Paciente J.G.S.C, sexo masculino, 09 anos de idade, compareceu acompanhado de sua responsável na clínica Odontológica do Centro Universitário Fametro para tratamento odontológico. Após a anamnese notou-se através do exame intra-oral que o paciente estava no período intertransitório da dentadura mista, ausência de apinhamentos e do elemento 55 com perda de espaço para a erupção do seu sucessor, (segundo pré-molar superior direito) devido a mesialização do elemento 16 caracterizando uma má-oclusão de classe II de Angle subdivisão direita (Figure 1). A responsável quando questionada a respeito da ausência do elemento dentário, afirmou que o elemento foi extraído precocemente em virtude de um processo carioso.

Figura 1 - A) Fotografia intrabucal lateral direita; B) fotografia intrabucal frontal; C) fotografia intrabucal lateral esquerda; D) fotografia intrabucal oclusal superior inicial; E) fotografia intrabucal oclusal inferior inicia.
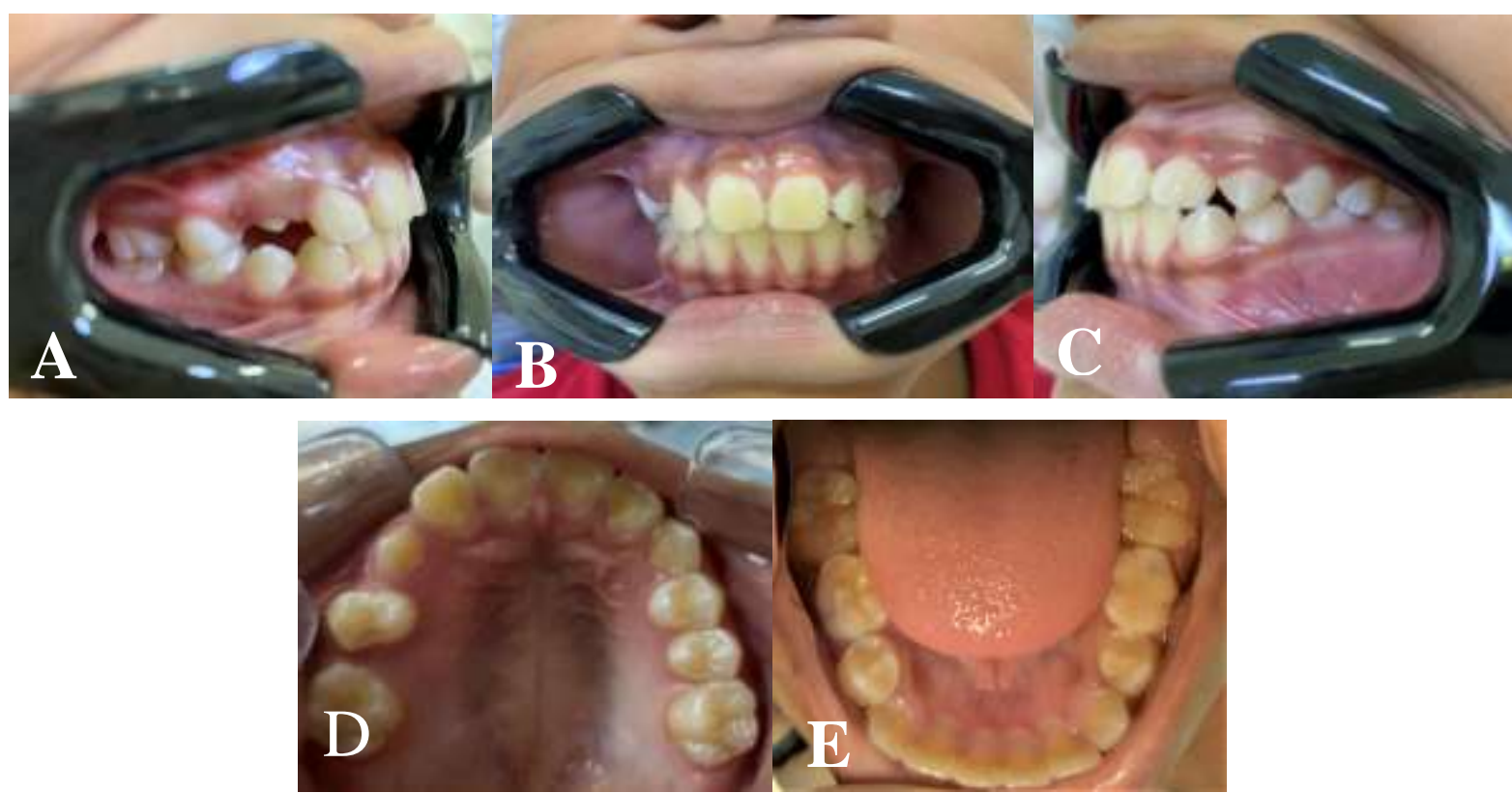

Fonte: Autores.

No exame radiográfico foi observado a presença do sucessor, com 1/3 de formação radicular correspondente ao estágio 7 de Nolla. Devido ainda a grande possibilidade de irrupção fisiológica, foi planejada a intervenção para a recuperação do espaço perdido (Figures 2). 
Figura 2 - Radiografia panorâmica inicial evidenciando o estágio de transição dentária e a perda precoce do elemento 55.

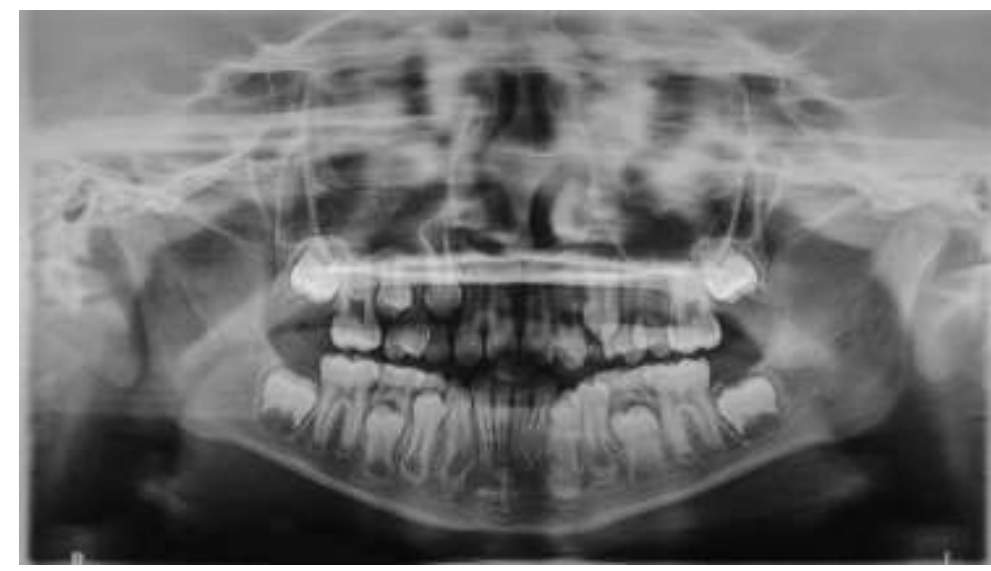

Fonte: Autores.

Após anamnese e exame clínico e radiográfico, foi planejado um aparelho para a recuperação do espaço dentário perdido. Sendo assim, optou-se pela instalação inicialmente de um dispositivo recuperador de espaço removível, composto por uma placa com torno expansor e uma mola digital para interceptação da má-oclusão (Figure 3).

Figura 3 - A) Fotografia intrabucal lateral direita com aparelho removível; B) fotografia intrabucal frontal com aparelho removível; C) fotografia intrabucal lateral esquerda com aparelho removível; D) fotografia intrabucal oclusal com aparelho, aparelho removível; E) fotografia do aparelho removível utilizado no caso inicialmente.

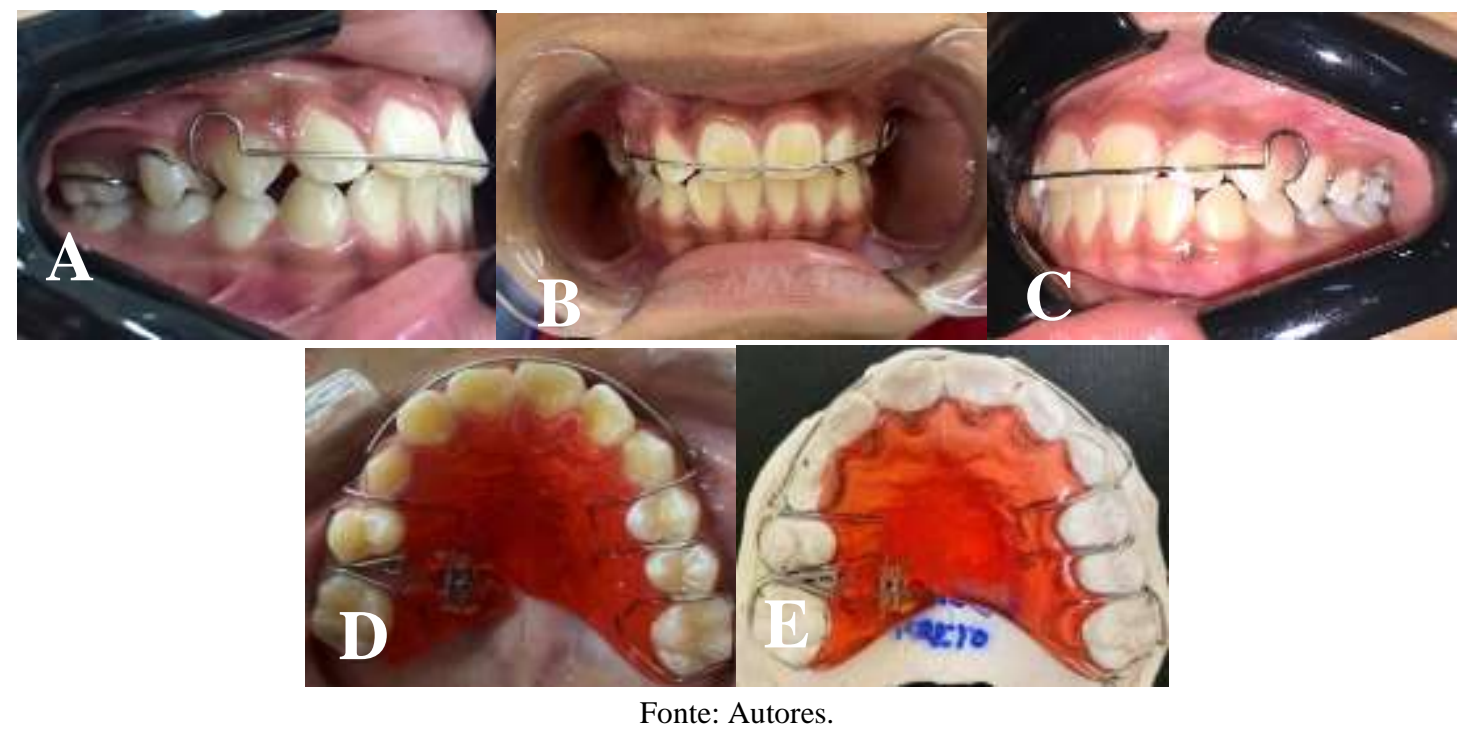

Para que o aparelho pudesse ser confeccionado, foi realizada uma moldagem anatômica do paciente com material hidrocolóide reversível a base de alginato Jeltrate tipo II Dentsply® (Dentsply Sirona Brasil, São Paulo/SP, Brasil). Em seguida, obteve-se os modelos de estudo e trabalho, que foram vazados com gesso tipo IV Asfer® (Asfer Indústria Química Ltda, Indaiatuba/SP, Brasil).

Posteriormente a confecção do aparelho, foi realizada a sua instalação com ativação inicial de 4/4 de volta do parafuso expansor. A primeira fase do tratamento durou 03 meses com ativações mensais na clínica, após esse período houve uma interrupção nos atendimentos clínicos na instituição, em decorrência da pandemia de Sars cov 2 . Durante 05 meses a responsável do paciente foi orientada a manter o aparelho em uso e recebeu orientações em como proceder com a higienização do mesmo em domicílio. Após retorno nos atendimentos clínicos, foi realizado 01 atendimento mensal com ativação de $4 / 4$ de 
volta e replanejamento do caso para a substituição do aparelho, já que durante o período de pausa, as ativações não foram realizadas. Dessa forma, o aparelho removível foi substituído por um aparelho fixo convencional, segmentado e associado a uma mola digital, a fim de acelerar a recuperação do espaço e aproveitar a maior força eruptiva do elemento dentário. A decisão de substituir o aparelho foi tomada a partir do acompanhamento clínico e radiográfico do elemento permanente que se encontrava com 2/3 de formação radicular, estágio 8 de Nolla, (Figura 4) (Katchburian et al., 2017).

Figura 4- A) Radiografia periapical evidenciando o estágio de formação radicular do elemento 15; B) aparelho fixo segmentado com mola digital instalado no paciente e irrupção inicial da ponta de cúspide do elemento 15.

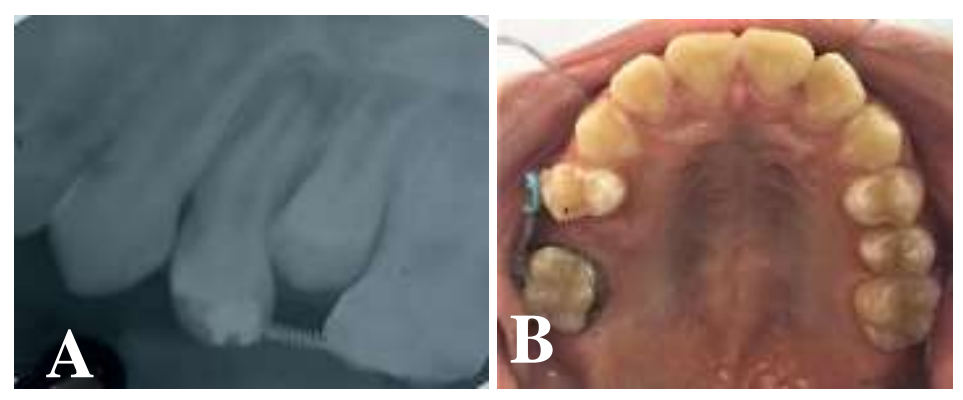

Fonte: Autores.

Para a instalação do aparelho fixo segmentado, foi necessário a cimentação de banda ortodôntica $\mathrm{n}^{\circ} 36 \mathrm{Morelli}^{\circledR}$ (Morelli Ortodontia, Sorocaba/SP, Brasil) com cimento de ionômero de vidro autopolimerizável de cimentação Maxxion C FGM® (FGM Dental Group, Joinville/SC, Brasil) no primeiro molar superior direito permanente. Apenas um bráquete da marca Abzil@, prescrição Roth, foi fixado com resina composta Opallis - FGM® (FGM Dental Group, Joinville/SC, Brasil) no elemento 14. A meta proposta para o tratamento foi alcançada após 3 meses com o aparelho fixo, onde pode-se observar a irrupção clínica das pontas de cúspides do pré-molar superior direito no espaço recuperado.

Após início do irrompimento do elemento dentário, o aparelho ficou como contenção por mais 02 meses, evitando movimentações dentárias e permitindo a completa irrupção do elemento 15, finalizando o caso com a remoção do aparelho. (Figura 5). 
Figura 5 - A) Fotografia intrabucal lateral direita final evidenciando a relação do molar de classe I de Angle; B) Fotografia intrabucal frontal final; C) fotografia intrabucal lateral esquerda; D) fotografia intrabucal oclusal superior evidenciando a correta erupção do elemento 15 ; E) fotografia intrabucal oclusal inferior final.

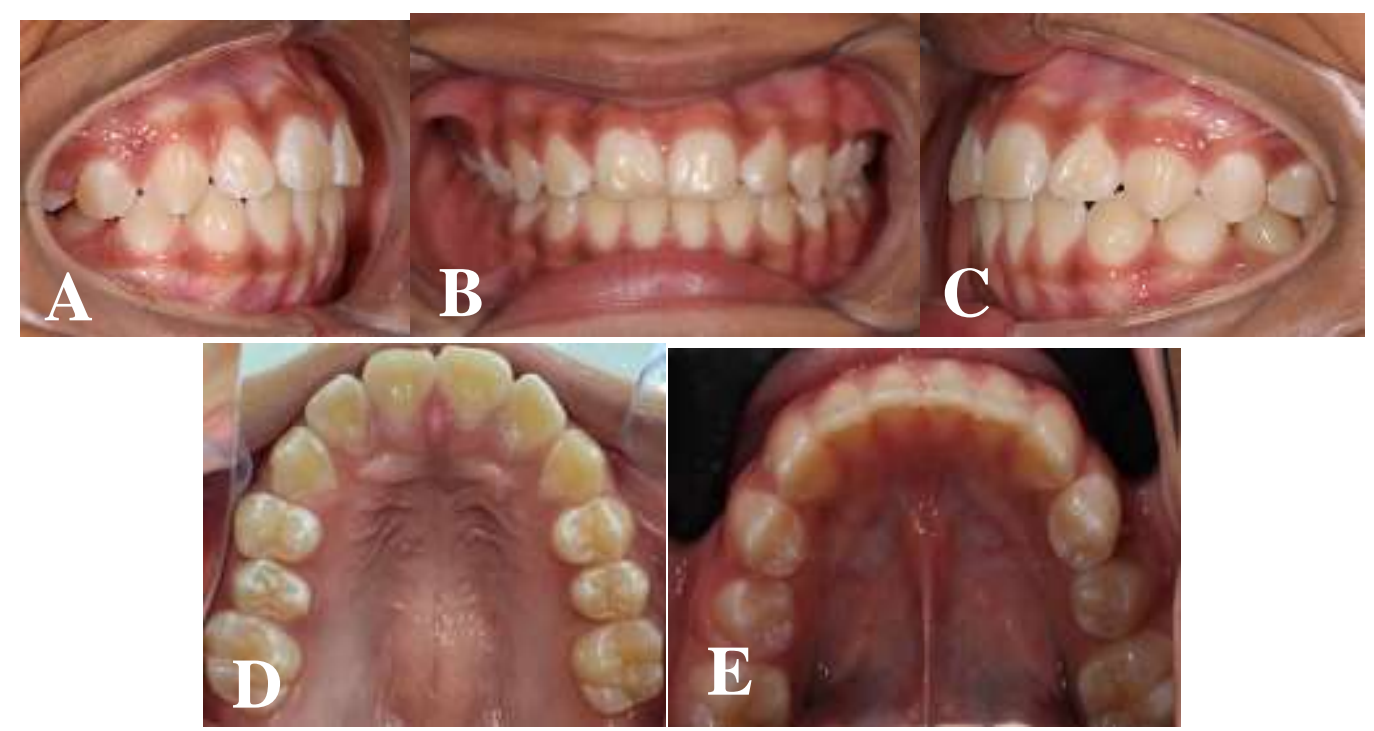

Fonte: Autores.

\section{Discussão}

No presente estudo, foi observado que a perda prematura de dentes decíduos pode apresentar como consequência o desequilíbrio da função mastigatória, desenvolvimento de más-oclusões e diminuição do espaço dentário, corroborando com outros autores que relatam ainda alterações na fonética e interferência no desenvolvimento craniofacial da criança (MonteSanto et al., 2018). Por isso a importância de manter a integridade desses dentes ou reabilitá-los em caso de perda.

O paciente apresentava alteração na relação chave-molar do lado correspondente a perda precoce, constatando assim, que a perda precoce de dentes decíduos pode induzir o surgimento de más-oclusões e interferir na erupção fisiológica do sucessor permanente, fato este já relatado por outro autor (Nobrega et al., 2018). As más-oclusões ocupam o terceiro lugar dentre as alterações bucais e podem ser tratadas através da ortodontia preventiva e interceptativa, que visa inibir desvios anormais dos padrões oclusais com a utilização de aparelhos (Morais et al., 2016; Lopes et al., 2019).

Conforme a literatura, o momento mais oportuno para a intervenção de uma má-oclusão oriunda de uma perda precoce, é durante a dentição mista, de tal maneira que favorece o desenvolvimento craniofacial e esquelético, previne disfunções temporomandibulares como também contribui para o bom posicionamento dos dentes permanentes (Silva et al., 2020). No trabalho desenvolvido, o paciente recebeu tratamento ortodôntico interceptativo ainda durante a dentadura mista, favorecendo a abertura de espaço para que o dente que estava impactado pudesse irromper, levando o molar para relação de normoclusão.

No estudo desenvolvido por Siqueira et al., enfatiza as desvantagens que o tratamento precoce apresenta, estando relacionadas na imprevisão do crescimento craniofacial, conhecimento da diversidade de eventos genéticos e ambientais que podem ter influência nesse processo, além de presciência da futura condição dos dentes permanentes por parte da equipe e/ou profissional responsável pelo tratamento (Siqueira et al., 20015).

Diante de situações de perdas precoces de dentes decíduos, os aparelhos ortodônticos como mantenedores de espaço podem ser utilizados para preservação do espaço até que o sucessor permanente inicie seu processo de irrupção fisiológica (Gomes et al., 2020; Watt et al., 2018). No presente caso, não havia indicação para a utilização de mantenedor de espaço, pois a má-oclusão já estava estabelecida, portanto, indicou-se o recuperador de espaço. 
Autores relatam que a escolha do aparelho é feita a partir do tipo de má-oclusão a ser tratada e deve respeitar a individualidade, cooperação e idade do paciente (Silva et al., 2020; Moreira et al., 2020). Por isso, optou-se inicialmente por um aparelho recuperador de espaço removível, visto que este aparelho atua na verticalização dos dentes vizinhos recuperando o espaço perdido, além de possibilitar higienização adequada, adaptações e ajustes conforme os resultados clínicos (Siqueira $e t$ al., 2015). No entanto, os resultados com o aparelho removível não foram satisfatórios, considerando que houve pausa no tratamento em decorrência da pandemia de Sars cov 2, sendo necessário substituí-lo por um aparelho fixo que apresentou resultado almejado a curto prazo.

\section{Conclusão}

Os recuperadores de espaço são aparelhos indicados para perda precoce de dentes decíduos. Portanto os recursos ofertados com o uso de aparelhos recuperadores de espaço são eficazes para interceptar e tratar más-oclusões diagnosticadas precocemente. No presente caso o resultado foi favorável com a distalização do molar e recuperação do espaço.

\section{Referências}

Amaral, R. C., Ferreira, I. A., Campello, S. C. S., Calvo, A. F. B., Tedesco, T. K., \& Imparato, J. C. P. (2019). Use of intrarradicular pins in primary teeth: case report of one year of accompanying. RGO, Rev Gaúch Odontol. 67, e20190041

Barbosa, V. L. T., Pierini, A. J., \& Gallo, Z. (2018). A prática da ortodontia na rede pública de saúde - uma revisão de literatura. Revista Brasileira Multidisciplinar, 21(1), 103-120.

Félez, I. N., Ângulo, L. M., Eguiluz, M. C., Vidal., C. C., Canela, M. C., Vitales, C. T., et al. (2020). Influencia de la nacionalidade en la prevalencia de enfermedades frecuentes em Atención Primaria. Rev Pediatr Aten Primaria.22, e1-11

Godoi, J., Silva, Y. A., Silva, W. S. L., Amorim, P. B., Marques, P. G., Quedevez, A. P., et al. (2019). Perda precoce do primeiro molar permanente. REAC/EJSC, 4, e729, https://doi.org/10.25248/reac.e729.2019

Gomes, G. V., Strelow, T. A. T., \& Almeida, S. A. (2020). Ortodontia Preventiva e Interceptativa e suas contribuições para um bom desenvolvimento da oclusão do paciente em fase de dentição decídua e/ou mista: um estudo teórico. J Business Techn, 14(2), 77.

Katchburian, E., \& Arana, V. (2017). Histologia e Embriologia Oral: texto, atlas-Correlações clínicas. Editorial Medica pan-americana. (4a ed.), Guanabara Koogan, 277.

Lopes, A. J. F., Santos, C. B., Ritzmann, M., Tiago, C. M., \& Nouer, P. R. A. (2019). Prevalência da maloclusão em escolares de 10 a 15 anos na cidade de Santo Antônio de Pádua - RJ. J Orof Invest. 6(2), 47-58.

López, S. E., D’Aquino, E. H., Cruces, F. W., Díaz T. V., \& Soza M. L. (2020). Determinantes de Caries Trempana de La Infancia em Niños em Riesgo Social. International Journal of Interdisciplinary Dentistry, 13(1), 26-29.

Macedo, A. G. O., Cunha, A. F., Cunha, A. C. P. P., Farias, A. C. R., Pereira, H. S. G. (2015). Projeto de Extensão: Educação Continuada em Ortodontia Preventiva e Interceptativa. Rev. Ciênc. Ext. 11(2), 70-78.

Monte-Santo, A. S., Viana, S. V. C., Moreira, K. M. S., Imparato, J. C. P., Mendes, F. M., \& Bonini, G. A. V. C. (2018). Prevalence of early loss of primary molar and its impact in schoolchildren's quality of life. Int J Paediatr Dent. 28(6), 595-601.

Morais, C. H., Zannin, L., Dega,n V. V., Valdrighi, H. C., Venezian, G. C, \& Vedovello S. A. S. (2016). Malocclusion in schoolhildren aged 7-12 years old in Minas Gerais, Brazil. RGO, Rev Gaúch Odontol, , 64(2), 164-170.

Moreira, A. K. S., Costa, G. C. B., Pantoja, J. K. M., \& Carlos, A. M. P. (2020). A Importância da Instalação de Mantenedor de Espaço Fixo Não Funcional em Odontopediatria - Revisão de Literatura. Braz. J. of Develop., 6(12), p. 97006-97015.

Moreno A. P. P., Aguiar A. P., Junior L. E. A., Crepaldi M. L. S., Sant'Ana, A. P., Crepaldi, A. A. (2018). Recuperação de espaços em dentadura mista com uso de aparelho hyrax, barra transpalatina e aparelho fixo 4x2. Revista FAIPE, 8(2), 8-20.

Neto, N. L., Cardoso, C. A. B., Abdo, R. C. C., \& Silva, S. M. B. (2016). Oral rehabilitation in pediatric dentistry: a clinical case report. RGO, Rev Gaúch Odontol, Porto Alegre, 64(1), 87-91.

Nobrega, M. L., Barbosa, C. C. N., \& Brum, S. C. (2018). Implicações da perda precoce em odontopediatria. Revista Pró-UniverSUS. 09(1), 61-67

Pereira, A. S., Shitsuka, D. M., Parreira, F. J., \& Shitsuka, R. (2018). Metodologia da pesquisa científica. UFSM.

Rosa, G. N., Buzzati, B. C. S., Piovesan, C., Mendes, F. M., Oliveira, M. D. M., Arrengui, T. M. (2015). Impact of malocclusion on oral health-related quality of life of preschool children. RGO, Rev Gaúch Odontol, , 63(1), 33-40. 
Research, Society and Development, v. 10, n. 17, e57101724514, 2021

(CC BY 4.0) | ISSN 2525-3409 | DOI: http://dx.doi.org/10.33448/rsd-v10i17.24514

Silva, D. B. H., \& Gonzaga, A. S. (2020). Importance of orthodontic intervention of the Class III malocclusion in mixed dentition. Dental Press J. Orthod. 25 (05). https://doi.org/10.1590/21776709.25.5.057-065.bbo

Silva, L. A. M. da ., Pinheiro, J. C., Silva, G. G. da ., Monteiro, F. A. B. da S., Porto, G. C. C., Silva, J. P. dos S., Rodrigues, D. dos A., Feitoza, V. A., Araujo, F. S., Moura, A. P. G. e., Santos, K. M. D. J., Silva, T. F. da ., Santos Junior, W. dos ., Andrade, A. S. de ., \& Silva, A. F. da . (2020). Utilização dos mantenedores e recuperadores de espaço na ortodontia interceptativa: Revisão dos conceitos atuais. Research, Society and Development, 9(11), e1009119627. https://doi.org/10.33448/rsd-v9i11.9627

Siqueira, M. A., Filho, I. J. Z., NaimKassis, E., \& Buchala, C. A. C. N. (2015). Preventive and Interceptative Orthodontics: review of its importance. Rev International Journal of Development Research 5(10),

Watt, E., Ahmad, A., Adamji, R., Katsimpali, A.., Ashley, P, \& Noar, J. (2018). Space maintainers in the primary and mixed dentition - a clinical guide. Br Dent J. 24;225(4):293-298. 10.1038/sj.bdj.2018.650. Erratum in: Br Dent J. Sep 28;225(6):555. 